\title{
Enhancements of Electric Machine Models: The EMachines Library
}

\author{
Anton Haumer ${ }^{1,2}$ Christian $\mathrm{Kral}^{2}$ \\ ${ }^{1}$ OTH Regensburg, Germany, anton . haumer@oth-regensburg.de \\ ${ }^{2}$ EDrives, Austria, \{anton.haumer, christian.kral \} dedrives.eu
}

\begin{abstract}
Transient models of multi phase electric machines are already implemented in the Modelica Standard Library (MSL). However, advanced effects like saturation and skin effect are not taken into account. As an extension to the MSL models, the new EMachines library is presented. This package will be released as a supplemental library to the commercial EDrives library. The particular focus of this paper is on the deep bar effect of induction machines. A comparison of simulation results demonstrates the influence of the skin effect on the operational behavior of the machines. At the end of this publication further developments of the EMachines library will be outlined.
\end{abstract}

Keywords: $\quad$ multi phase electric machines, induction machines, squirrel cage, deep bar effect, skin effect

\section{Introduction}

The Modelica Standard Library already contains transient models of multi phase electric machines:

Modelica.Electrical.Machines and

Modelica.Magnetic.FundamentalWave (Kral,

Haumer, Wöhrnschimmel, 2014). For the next release of the MSL, quasi static machine models are planned to be included. These models neglect electric transients for performance reasons (Kral, Haumer, 2014):

Modelica.Magnetic.QuasiStaticFundamentalWave.

Both the transient and the quasi static models consider Joule, friction, core and stray load losses. However, more advanced effects like saturation and skin effect are not taken into account. During the development of the EDrives Library (Haumer, Kral, 2014) and the extension towards controlled multiphase operation, the stator stray inductance $L_{s \sigma}$ design had to be changed. Therefore, in the EMachines library the stator stray inductances are modeled by phase inductances with mutual coupling to other phases and by self inductances - ideally coupled with the respective phase only. The new leakage inductance concept was implemented in the electric machine models of the EMachines library without affecting the backwards compatibility of the machine parameters. Yet the new machine models are based on a parameter record to overcome the drawback of parameterization by multiple parameters. In the new EMachines Library advanced effects like saturation and skin effect will be taken into account. First, the deep bar effect is implemented, followed by further effects.

The EMachines library will be released as a supplemental library to the commercial EDrives library (see Table 1). Yet the EMachines library can be used without the EDrives library to investigate the influence of advanced effects of machines fed directly from the supply network.

Table 1. Description of electric drives libraries

\begin{tabular}{|c|c|}
\hline library & description \\
\hline $\begin{array}{l}\text { Modelica. } \\
\text { Electrical. } \\
\text { Machines }\end{array}$ & $\begin{array}{l}\text { transient threephase models, } \\
\text { based on space phasor theory, } \\
\text { connector and parameter } \\
\text { compatible } \\
\text { Magnetic. FundamentalWave }\end{array}$ \\
\hline $\begin{array}{l}\text { Modelica. } \\
\text { Magnetic. } \\
\text { FundamentalWave }\end{array}$ & $\begin{array}{l}\text { transient multiphase } \text { models, } \\
\text { based on coupling between } \\
\text { electrical domain } \\
\text { fundamental magnetic field }\end{array}$ \\
\hline $\begin{array}{l}\text { Modelica. } \\
\text { Magnetic. } \\
\text { Quasistatic. } \\
\text { FundamentalWave }\end{array}$ & $\begin{array}{l}\text { quasi static multiphase models, } \\
\text { based on coupling between } \\
\text { electrical domain and } \\
\text { fundamental magnetic field, } \\
\text { neglecting electrical transients } \\
\text { based on time phasors }\end{array}$ \\
\hline EMachines & $\begin{array}{l}\text { supplement to the commercial } \\
\text { EDrives library, extending } \\
\text { Magnetic.FundamentalWave } \\
\text { and Magnetic.Quasistatic. } \\
\text { FundamentalWave }\end{array}$ \\
\hline EDrives & $\begin{array}{l}\text { commercial library for inverter } \\
\text { fed drives, utilizing models from } \\
\text { EMachines }\end{array}$ \\
\hline
\end{tabular}

First, the structure of the EMachines library (see Figure 1) will be presented, including the parameterization by means of parameter records. Second, the technical details of the deep bar effect will be explained. Comparisons of simulation results demonstrate the handling of the machine models and the influence of the skin effect. At the end of this paper an overview on the future developments of the EMachines library will be given. 


\section{Structure of the Library}

\begin{tabular}{|c|}
\hline EMachines \\
\hline (i) User's Guide \\
\hline Examples \\
\hline ParameterRecords \\
\hline 田SmpmData \\
\hline "⿴囗十⺀SmrData \\
\hline 曲ImcData \\
\hline$\stackrel{+}{+} \cdot \square$ Components \\
\hline$\pm \cdot \circ$ BaseClasses \\
\hline QuasiStatic \\
\hline BasicMachines \\
\hline SynchronousMachines \\
\hline InductionMachines \\
\hline : $\square$ Components \\
\hline +. BaseClasses \\
\hline Transient \\
\hline : BasicMachines \\
\hline : SynchronousMachines \\
\hline InductionMachines \\
\hline$\oplus \cdot \square$ Components \\
\hline$\pm \cdot 0$ BaseClasses \\
\hline$+i$ Icons \\
\hline
\end{tabular}

Figure 1. Structure of the EMachines Library

The EMachines library includes both transient and quasi static models of synchronous and induction machines. The EMachines models reuse the components of the Modelica.Magnetic.FundamentalWave and the Modelica.Magnetic.QuasiStaticFundamentalWave

library and are thus fully connector compatible. Even though the parameters of the MSL library are also used by the EMachines models, the EMachines library is not parameter compatible since a parameter record is used (see Figure 2). This concept allows the user to switch from the parameter set of one drive to another parameter set in a very convenient way. All parameters that do not specify the machine properties but operational conditions remain single parameters in the machine models (see Figure 3). These single parameters are operational temperatures in case of a disabled heat port of the machine, and the enabling parameter of the optional support flange.

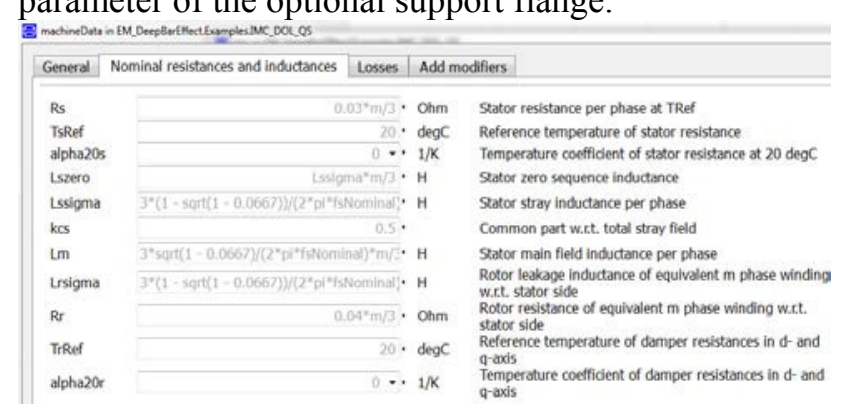

Figure 2. Parameter record of the induction machine

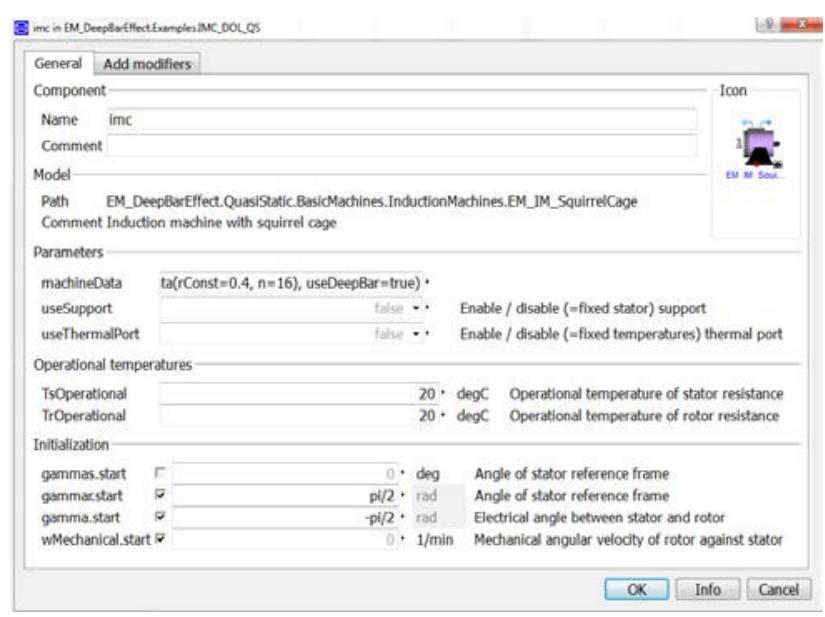

Figure 3. Operational parameters of induction machine

Components for modeling enhanced effects are stored in sub-packages named components. The parameter records of these components and the entire machines are defined in the package ParameterRecords. Extensions are implemented in such a way that the user can switch them off or on. This concept enables the convenient comparison of the operational behavior of standard and enhanced electric machine models.

The EDrives library uses wrapper models. In these wrapper models the instances of EMachines models are used, including temperature and rotor position sensors. The wrapper models also use a signal bus connector to exchange the sensed quantities with the inverter models (Haumer, Kral, 2014).

Up to now, the following machine types were implemented:

- Synchronous machine with permanent magnets

- Synchronous reluctance machine and

- Induction machine with squirrel cage rotor.

Further machine types (electrically excited synchronous machine, induction machine with wound rotor and slip rings) will be implemented in the near future.

\section{Deep Bar Effect}

The skin effect in the bars of the squirrel cages of induction machines and the damper cages of synchronous machines is called deep bar effect (Binder, 2012; Toliyat, Kliman, 2004). Skin effect in general is caused by the linkage between electric and magnetic field, it describes the fact that current density increases from the center of a conductor to its surface, dependent on the current's frequency. In this paper it is assumed that the bar width is smaller than the height. Considering the distribution of the stray field (Kleinrath, 1975), it is sufficient to only consider a one dimensional model of the skin effect. The deep bar effect increases the effective resistance and decreases the effective stray inductance of the rotor bars. This effect strongly depends on the electrical rotor 
frequency. Therefore the stator current and torque of induction machines at stand still, i.e. slip $=1$, is strongly affected. Furthermore, the additional losses caused by higher harmonics of non-sinusoidal currents due to inverter operation are increased. Modeling the deep bar effect allows the investigation of the starting behavior of induction machines fed by the grid in a more realistic way. For inverter fed machines, the influence of resistance increase and stray inductance decrease on the inverter's controller can be investigated.

\subsection{Differential equations}

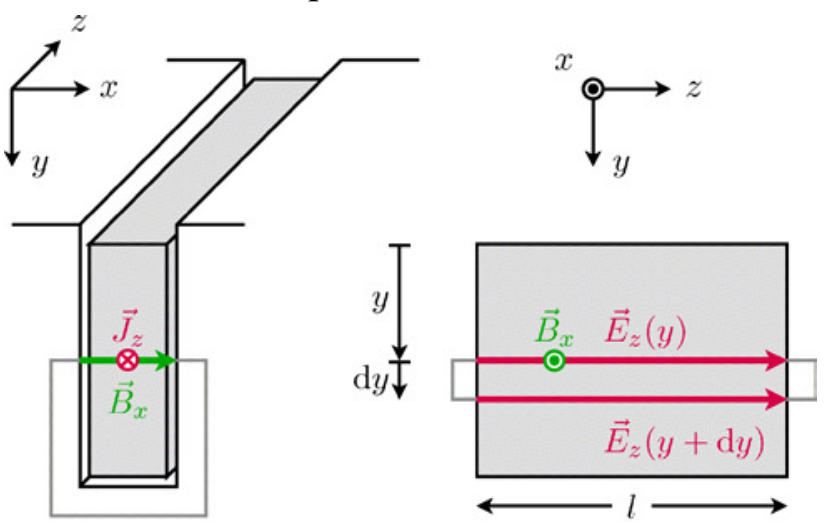

Figure 4. Deep bar embedded in rotor sheets

In Figure 4, one can relate the magnetic stray field strength $H_{x}$, and the magnetic flux density $B_{x}$,

$$
\vec{B}_{x}=\mu_{0} \vec{H}_{x}
$$

respectively, with the current density $J_{z}$. The magnetic permeability of the iron sheets is considered to be infinitely high:

$$
\vec{H}_{x}(y) b(y)=\int_{y}^{h} \vec{J}_{z}\left(y^{\prime}\right) b\left(y^{\prime}\right) d y^{\prime}
$$

where $h$ designates the total height of the bar.

The magnetic flux in $x$-direction of a layer of infinitely small height can be expressed as

$$
\mathrm{d} \phi=\mu_{0} H_{x}(y) l d y=\mu_{0} \frac{l d y}{b(y)} \int_{y}^{h} J_{z}\left(y^{\prime}\right) b\left(y^{\prime}\right) d y^{\prime}
$$

where $l$ is the conductor length. If the bar current and therefore the bar current density is varying with respect to time, the time varying flux induces a voltage in the bar in z-direction, which in turn influences the current flow

$$
\begin{gathered}
\oint \vec{E} \cdot \overrightarrow{d s}=\left[E_{z}(y+d y)-E_{z}(y)\right] l=-\frac{\partial(\mathrm{d} \phi)}{\partial t} \\
\kappa E_{z}(y)=J_{z}(y)
\end{gathered}
$$

In (5) $\kappa$ represents the electric resistivity. The current flow caused by the induced voltage reduces the current density for greater $y$ (i.e. towards the slot ground) and increases the current density for smaller $y$ values (i.e. towards the slot opening). The partial differential equation for the transient skin effect yields:

$$
\frac{\partial^{2} E_{z}}{\partial y^{2}}=\mu_{0} \kappa \frac{\partial E_{y}}{\partial t}
$$

Assuming a sinusoidal current with constant frequency, and utilizing space phasors to express the sinusoidal quantities (Haumer et al, 2008), the partial differential equation simplifies to an ordinary differential equation with respect to the height coordinate y:

$$
\frac{d^{2} \underline{E}_{z}}{d y^{2}}=j \omega \mu_{0} \kappa \underline{E}_{z}
$$

For a rectangular bar the solution of (7) leads to an exponential distribution of the current density:

$$
\underline{J}_{z}=J_{0} e^{-(1+j) \frac{y}{\delta}}
$$

where $\delta=\sqrt{\frac{2}{\omega \mu_{0} \kappa}}$ denotes the skin depth and $J_{0}$ represents the current density at the top of the bar.

Considering a rectangular bar made of aluminum with a height of $30 \mathrm{~mm}$ and a width of $4 \mathrm{~mm}$, the current densities with respect to height are depicted in Figure 5 for three different frequencies. The total bar current in all cases gives $120 \mathrm{~A}$.

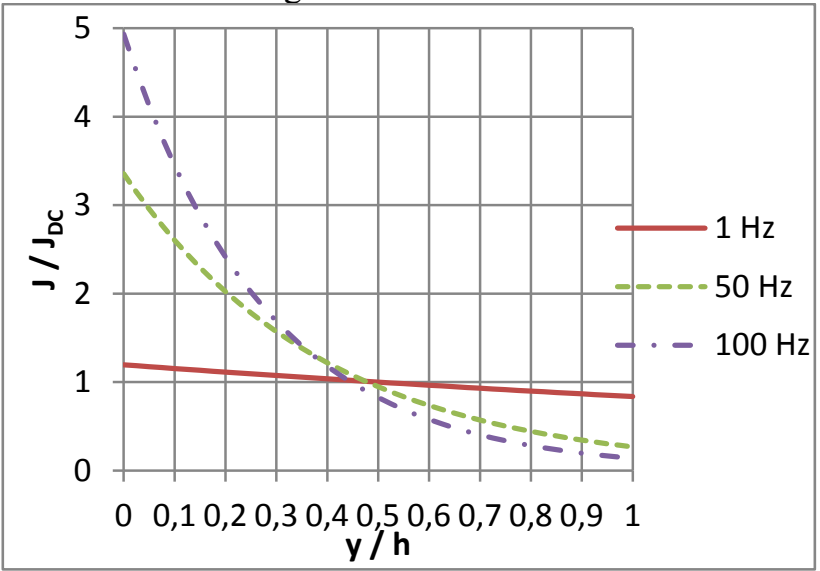

Figure 5. Current densities with respect to height

For simple geometries like rectangular or trapezoidal bars (zur Nieden, 1931), the differential equation (7) can be solved analytically. It is then possible to express the frequency dependent factors of resistance increase, $k_{R}$, and stray inductance decrease, $k_{L}$, with respect to the DC resistance and leakage inductance, respectively. For bars with rectangular shape, these factors are well known as Field's formulas, utilizing the so-called reduced height of the bar $\xi$ :

$$
\begin{gathered}
\xi(\omega)=h \sqrt{\omega \frac{\mu_{0} \kappa}{2}} \\
k_{R}=\xi \frac{\sinh (2 \xi)+\sin (2 \xi)}{\cosh (2 \xi)-\cos (2 \xi)} \\
k_{L}=\frac{3}{2 \xi} \frac{\sinh (2 \xi)-\sin (2 \xi)}{\cosh (2 \xi)-\cos (2 \xi)}
\end{gathered}
$$


To cope with arbitrary bar shapes, the height of the bar is discretized as described in (Müller et al, 2008). The rotor resistance $R_{R}$ and rotor stray inductance $L_{R \sigma}$ are separated into the constant parts $R_{R, c o n}$ and $L_{R \sigma, c o n}$ not affected by the skin effect and the variable parts $R_{R, v a r}$ and $L_{R \sigma, v a r}$ of the bar. The series connection of the variable part $R_{R, v a r}$ and $L_{R \sigma, v a r}$ is either replaced by a ladder network where each L-R element represents a layer of the discretized height, or the algorithm described in section 3.3 is evaluated. However, this algorithm can be applied only for quasi static points of operation: for transient operation, (6) has to be solved, using a ladder network (section 3.4).

The original cage used in Modelica.Magnetic.FundamentalWave and Modelica.Magnetic. QuasiStaticFundamentalWave is replaced by a new cage model shown in Figure 6 . The sub-model deepBar represents the variable parts of the rotor resistance $R_{R, v a r}$ and stray inductance $L_{R \sigma, v a r}$ under influence of the deep bar effect, whereas the constant parts of the rotor resistance and stray inductance is represented by the single components resistorconst and inductorConst. For performance reasons, the quasi static cage model utilizes the algorithm described in section 3.3, whereas the transient cage model uses the ladder network of section 3.4. The usage of the quasi static algorithm increases performance due to the fact that the algebraic equations can be solved easier than the set of differential equations describing the ladder network.

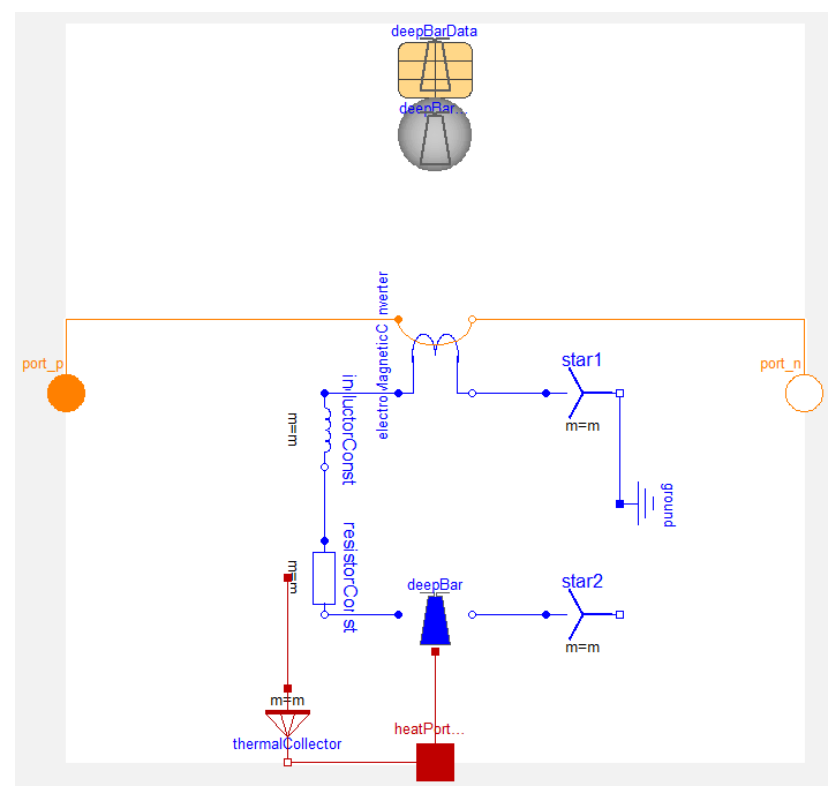

Figure 6. Transient cage model with skin effect

\subsection{Parameterization of the Cage}

For both - the quasi static algorithm and the transient ladder network - the resistances and stray inductances of the layers are pre-calculated in the parameter record of the cage: the user has to define the geometry of the bar (i.e. a matrix with the height coordinate $y$ in the first column and the respective bar width in the second column). An algorithm calculates the height $h_{k}$ and the width $b_{k}$ of each of the $n$ layers. From these heights and widths, the resistances $R_{k}$ at reference temperature and inductances $L_{k}$ of each layer are determined:

$$
\begin{aligned}
R_{k} & =\frac{l}{\kappa} \frac{1}{h_{k} b_{k}} \\
L_{k} & =\mu_{0} l \frac{h_{k}}{b_{k}}
\end{aligned}
$$

For the actual resistances the operating temperature has to be considered.

The user has to define the constant part $R_{R, c o n}$ of the rotor resistance with respect to the stator winding. Thus the variable part $R_{R, v a r}=R_{R}-R_{R, c o n}$ is determined, too. Since the geometry of the bar defines the area of cross section and therefore the DC resistance of a single bar, $R_{\text {bar }}$, the turns ratio between stator winding and a rotor bar is given:

$$
\text { turnsRatio }^{2}=\frac{R_{R, v a r}}{R_{\text {bar }}}
$$

The geometry defines the DC slot stray inductance $L_{b a r}$, too. Therefore the variable and the constant, $L_{R \sigma, v a r}$ and $L_{R \sigma, c o n}$, respectively, are determined by:

$$
\begin{gathered}
L_{R \sigma, v a r}=\text { turnsRatio }{ }^{2} L_{b a r} \\
L_{R \sigma, \text { con }}=L_{R \sigma}-L_{R \sigma, v a r}
\end{gathered}
$$

\subsection{Quasi static Algorithm}

In order to determine the actual resistance and stray inductance, we may assume the complex current in the first layer at the bottom of the bar, and recursively calculate one layer current after another, utilizing the Modelica definition of complex numbers:

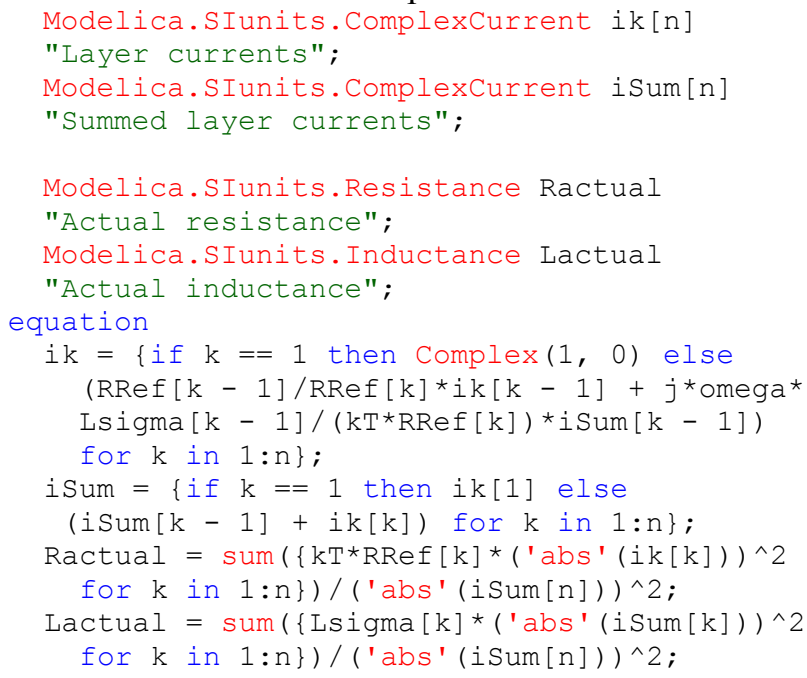

In this algorithm $\operatorname{RRe}[k]$ represents the reference resistance of each layer and $\mathrm{kT}$ is the relative resistance increase at operating temperature compared to reference temperature. The total resistance and inductance calculations are based on the linear equivalent circuit shown in Figure 7. 
Based on the actual resistance and stray inductance the actual voltage drop and Joule losses of the bar are calculated.

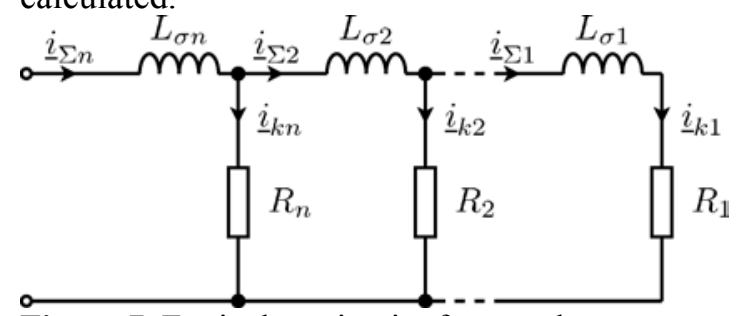

Figure 7. Equivalent circuit of a rotor bar

\subsection{Transient Equivalent Ladder Network}

In the transient deepBar model, a physical series connection of $n$ (count of layers) R-L elements is established; The entire network structure is depicted in Figure 7 and a single R-L element is shown in Figure 8. The single R-L elements are connected in series.

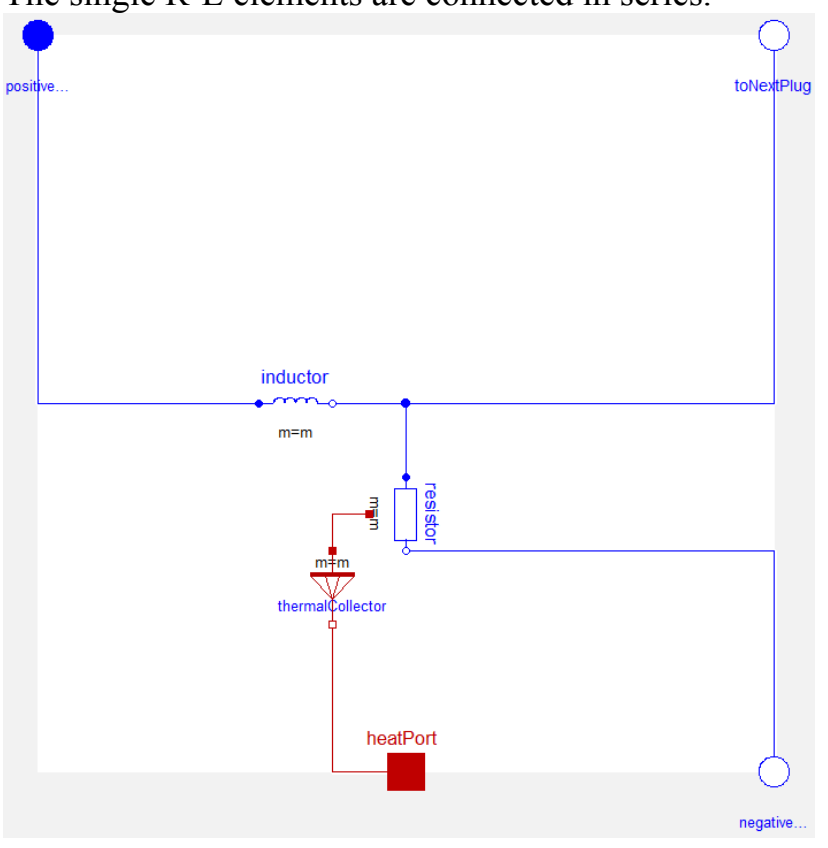

Figure 8. Transient deepBar model

\section{Simulation Results}

First, we compare the frequency dependent resistance increase $k_{R}$ and stray inductance decrease $k_{L}$ for a trapezoidal bar. For this bar shape the quasi static and the transient simulation and the analytically derived result (zur Nieden, 1931) are compared.

Figure 9 shows the quasi static model to evaluate the deep bar algorithm. The transient model looks similar; the frequency ramp raises very slowly from nearly 0 to $100 \mathrm{~Hz}$ to avoid transient effects as far as possible. Current and voltage are measured to calculate the effective impedance. The parameters of the investigated bar are summarized in Table 2. The bar is discretized into 16 layers.

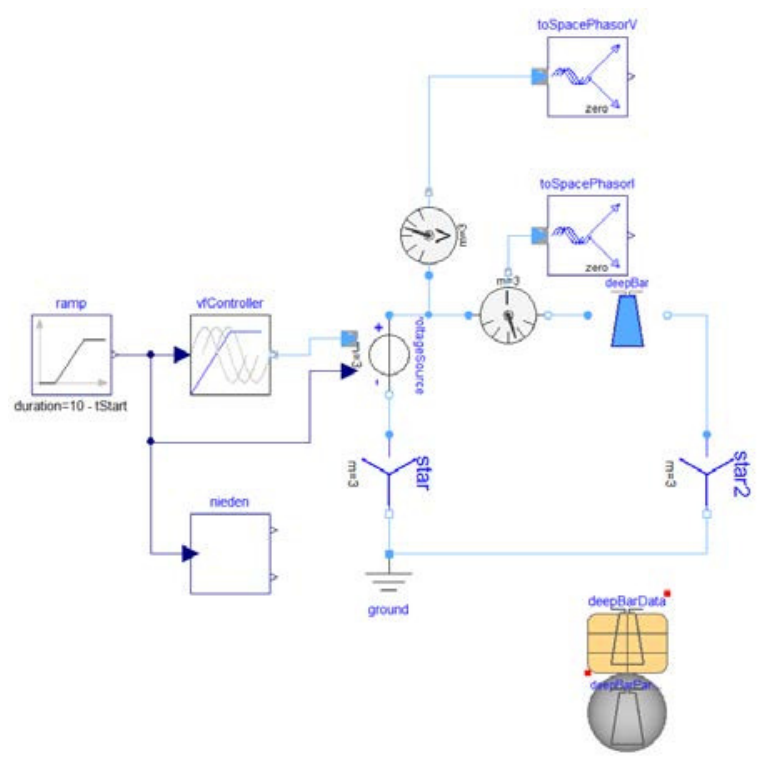

Figure 9. Quasi static evaluation of deep bar algorithm

Table 2. Parameters of the investigated bar

\begin{tabular}{|l|c|l|}
\hline height of bar & 30 & $\mathrm{~mm}$ \\
\hline top width & 6 & $\mathrm{~mm}$ \\
\hline bottom width & 2 & $\mathrm{~mm}$ \\
\hline conductivity & $36 \cdot 10^{6}$ & $\mathrm{~S} / \mathrm{m}$ at $20^{\circ} \mathrm{C}$ \\
\hline
\end{tabular}

Both the quasi static and the transient model calculate the resistance increase, $k_{R}$, and stray inductance decrease, $k_{L}$, from the actual Joule losses and energy of the magnetic field. The results of these calculations are summarized in Table 3 and Figure 10.

The transient model shows initial transients caused by the layer currents penetrating the bar. However, both the quasi static and the transient model match very well. Since the number of layers was chosen relatively low, there are deviations from the analytically obtained results. An appropriate choice of the count of layers leads to a trade-off between performance and accuracy of the results.

Table 3. Resistance increase and inductance decrease

\begin{tabular}{|r|r|r|r|r|r|r|}
\cline { 2 - 7 } \multicolumn{1}{c|}{} & \multicolumn{2}{c|}{ analytical } & \multicolumn{2}{c|}{ quasi static } & \multicolumn{2}{c|}{ transient } \\
\hline $\mathrm{f} / \mathrm{Hz}$ & $\mathrm{kR}$ & $\mathrm{kX}$ & $\mathrm{kR}$ & $\mathrm{kX}$ & $\mathrm{kR}$ & $\mathrm{kX}$ \\
\hline 1 & 1,001 & 1,000 & 1,001 & 1,000 & 1,000 & 0,882 \\
\hline 10 & 1,072 & 0,969 & 1,080 & 0,969 & 1,075 & 0,964 \\
\hline 20 & 1,266 & 0,891 & 1,276 & 0,894 & 1,273 & 0,894 \\
\hline 30 & 1,505 & 0,797 & 1,509 & 0,808 & 1,508 & 0,808 \\
\hline 40 & 1,731 & 0,711 & 1,731 & 0,730 & 1,730 & 0,730 \\
\hline 50 & 1,929 & 0,640 & 1,926 & 0,667 & 1,925 & 0,667 \\
\hline 60 & 2,100 & 0,584 & 2,095 & 0,618 & 2,095 & 0,618 \\
\hline 70 & 2,251 & 0,540 & 2,245 & 0,579 & 2,245 & 0,579 \\
\hline 80 & 2,388 & 0,504 & 2,381 & 0,547 & 2,380 & 0,547 \\
\hline 90 & 2,515 & 0,475 & 2,506 & 0,522 & 2,506 & 0,522 \\
\hline 100 & 2,635 & 0,451 & 2,623 & 0,500 & 2,623 & 0,500 \\
\hline
\end{tabular}




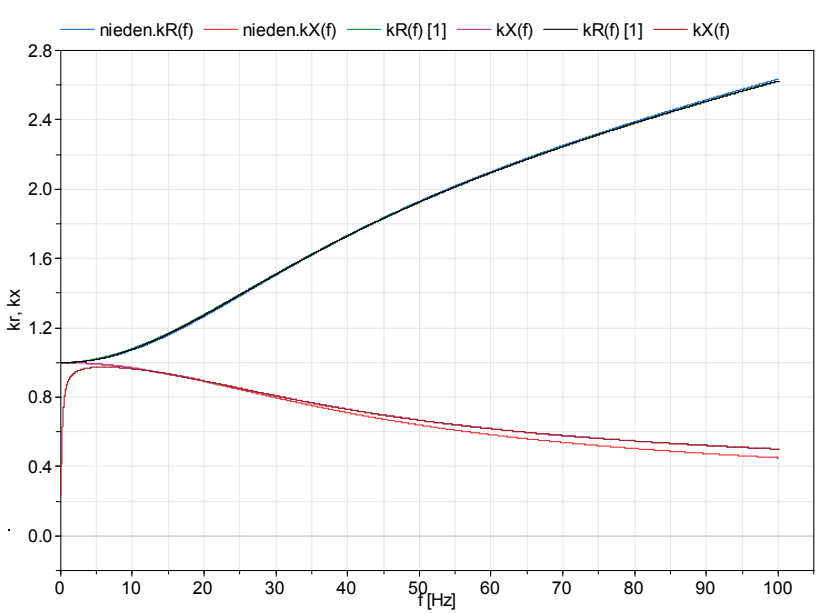

Figure 10. Quasi static (green and magenta) and transient (black and brown) results, compared with analytical formulae (blue and red)

The second example is derived from

Modelica.Electrical. Machines.Examples.

AsynchronousInductionMachines.AIMC_DOI

and simulates the start-up of an induction machine with squirrel cage, both quasi static and transient. Both models have the same diagram layout; the transient model is shown in Figure 11. Each induction machine model is loaded with a quadratic speed dependent torque.

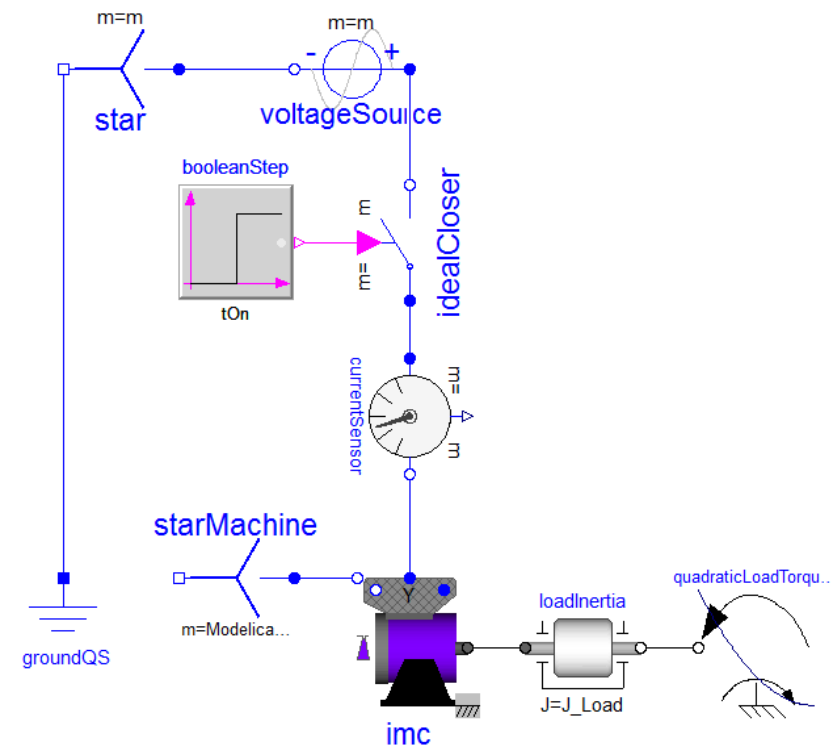

Figure 11. Transient start-up of induction machine

For the investigated machine the default data of the MSL induction machines are used; for the bar, the dimensions described in Table 2 are utilized.

Figure 12 shows the electromagnetic torque without and with deep bar effect of a quasi static simulation. Figure 13 shows the electromagnetic torque without and with deep bar effect of a transient simulation. Obviously, the deep bar effect causes a higher torque and reduces the duration of acceleration.

In each of the investigated cases the equilibrium at the end of the acceleration is practically the same, since for small slip (i.e. rotor frequency) the skin effect can be neglected.

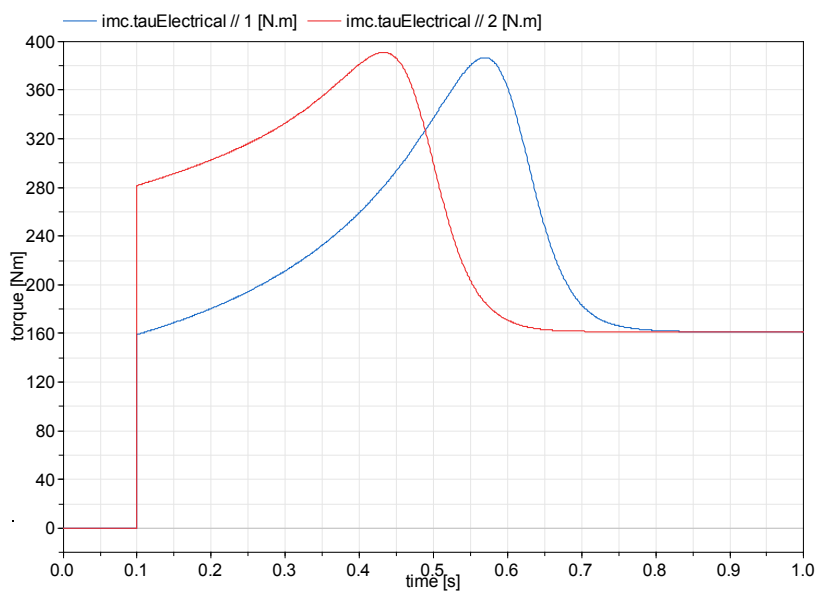

Figure 12. Quasi static torque w/o (blue) and with (red) skin effect

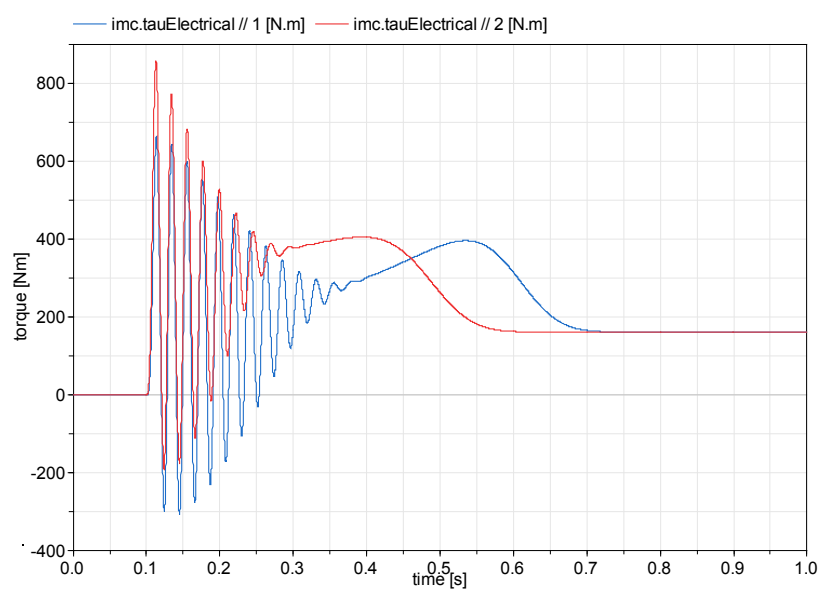

Figure 13. Transient torque w/o (blue) and with (red) skin effect

\section{Conclusions and Outlook}

The new EMachines library will be released as a supplemental library to the commercial EDrives library. The advantages and extensions to MSL models have been presented, especially the convenient parameterization of machine models by means of a parameter record.

In detail, the skin effect and its implementation have been discussed. A comparison of numerical and analytical factors of quasi static resistance increase and inductance decrease show satisfying coincidence. An example demonstrates the influence of the deep bar effect on the starting behavior of an induction machine.

All models are documented thoroughly and have been tested with both OpenModelica and Dymola. Examples demonstrating the usage are available. 
The EMachines library is a perfect platform for extensions of machine models in the near future without losing backwards compatibility to the MSL. Further developments are already planned:

- Induction machine models with wound rotor and slip rings

- Electrically excited synchronous machines

- Saturation effect of the main field and of the stray field

- Thermal models of electric machines

- Temperature dependent characteristic of permanent magnet

- Effect of cross-coupling between d- and q-axis in synchronous machines

- Effects of higher harmonics of the spatial distribution of the magnetic field

- Hysteresis core losses

- Detailed losses in the permanent magnet of permanent magnet synchronous machines

- Coupling with FEA software in order to take into account the detailed magnetic operation point

\section{References}

Andreas Binder. Elektrische Maschinen und Antriebe. Springer, 2012. doi: 10.1007/978-3-540-71850-5

H. A. Toliyat, G. B. Kliman. Handbook of Electrical Motors. CRC Press, 2004. ISBN 978-0824741051

Hans Kleinrath. Grundlagen elektrischer Maschinen. Akademische Verlagsgesellschaft 1975. ISBN 3-400-00279-8 (out of print).

Anton Haumer, Christian Kral, Johannes Vinzenz Gragger, Hansjörg Kapeller. Quasi-Stationary Modeling and Simulation of Electrical Circuits using Complex Phasors. Modelica 2008.

Anton Haumer, Christian Kral. The New EDrives Library: A Modular Tool for Engineering of Electric Drives. Modelica 2014.

Christian Kral, Anton Haumer, Reinhard Wöhrnschimmel. Extension of the FundamentalWave Library towards Multi Phase Electric Machine Models. Modelica 2014.

Christian Kral, Anton Haumer. New Multi Phase Quasi Static FundamentalWave Electric Machine Models for High Performance Simulations. Modelica 2014(a).

Germar Müller, Karl Vogt, Bernd Ponick. Berechnung elektrischer Maschinen. Wiley, 2008.

E. zur Nieden. Berechnung von Stäben für Stromverdrängungsmotoren. Elektrotechnik und Maschinenbau 51 (11): 129-134, 1931. 